\title{
Cycle-Accurate Evaluation of Software-Hardware Co-Design of Decimal Computation in RISC-V Ecosystem
}

\author{
Riaz-ul-haque Mian \\ Graduate School of Information Science \\ Nara Institute of Science and Technology \\ Ikoma, Japan \\ mian.riaz-ul-haque.mn3@is.naist.jp
}

\author{
Michihiro Shintani and Michiko Inoue \\ Graduate School of Science and Technology \\ Nara Institute of Science and Technology \\ Ikoma, Japan \\ $\{$ shintani,kounoe\}@is.naist.jp
}

\begin{abstract}
Software-hardware co-design solutions for decimal computation can provide several Pareto points to development of embedded systems in terms of hardware cost and performance. This paper demonstrates how to accurately evaluate such co-design solutions using RISC-V ecosystem. In a softwarehardware co-design solution, a part of solution requires dedicated hardware. In our evaluation framework, we develop new decimal oriented instructions supported by an accelerator. The framework can realize cycle-accurate analysis for performance as well as hardware overhead for co-design solutions for decimal computation. The obtained performance result is compared with an estimation with dummy functions.
\end{abstract}

Index Terms-RISC-V, RoCC, Hardware accelerator, Rocket chip, Decimal arithmetic, Decimal multiplication, Evaluation framework

\section{INTRODUCTION}

Decimal arithmetic is widely used in financial and scientific applications. Thus, IEEE 754 (Standard for floating-point arithmetic) has been revised to include decimal floating-point formats and operations [1]. Many software (SW) languages support decimal arithmetic that is realized with binary hardware units. However, these may not be satisfactory for a very large application in terms of performance. Many financial applications need to keep the quality of their customer service concurrently with the back-end computing process where computing time is a matter for the business owner.

The decimal arithmetic can be computed with software (arithmetic with binary hardware units) [2]-[4], hardware (dedicated hardware unit for decimal floating-point arithmetic) [5]-[8], or combination of both [9]. Software solutions are flexible and no additional hardware cost is involved. Hardware solutions require high-performance dedicated decimal units with high hardware cost. Software-hardware codesign solutions can co-optimize flexibility, performance and hardware cost and give several Pareto points to development of embedded systems. In software-hardware co-design solutions, a part of solution requires some dedicated hardware while other part can be executed in standard processors supporting binary arithmetics. However, evaluation of co-design solutions requires special evaluation environments.

In [9], four software-hardware co-design methods for decimal multiplication are proposed. part. A software part is evaluated by running it in several software platforms by replacing hardware part with dummy functions, while a hardware part is evaluated by designing hardware with computeraided-design tools. The environment can roughly evaluate the total performance as an execution time of software program with dummy functions.

To obtain more accurate evaluation, integrated environment with dedicated hardware design, software platform, and the interface between them is required. An open-source processor like UltraSparc T2 architecture [10] from Oracle/Sun (the first 64-bit microprocessors open-sourced) with standard SPARC instruction set architecture [11] can be used for such evaluation. However, it requires not only adding new decimal floating-point units and new instructions for them but also software tools to generate and simulate binary codes for the new architecture. SPARC V9 architecture provides IMPDEP1, 2 (Implementation-Dependent Instruction 1,2) and they can be used for new custom instructions.

In this work, we develop an evaluation framework for software-hardware co-design of decimal computation using RISC-V ecosystem [12]. RISC-V ecosystem is an opensource environment including RISC-V ISA, Rocket chip (one hardware implementation for RISC-V), RoCC (Rocket custom co-processor, Rocket chip interface to support accelerators), several languages for software and hardware, and several tools for verification and evaluation. In the proposed framework, a co-design solution is realized as a software that accepts new decimal-oriented instructions, and the new instructions are supported by a dedicated accelerator. Cycle-accurate analysis is given by emulating RISC-V binary on Rocket chip with the dedicated accelerator.

The key contribution of this paper is listed below:

1) Development of an evaluation framework for softwarehardware co-design solutions of decimal computation. 


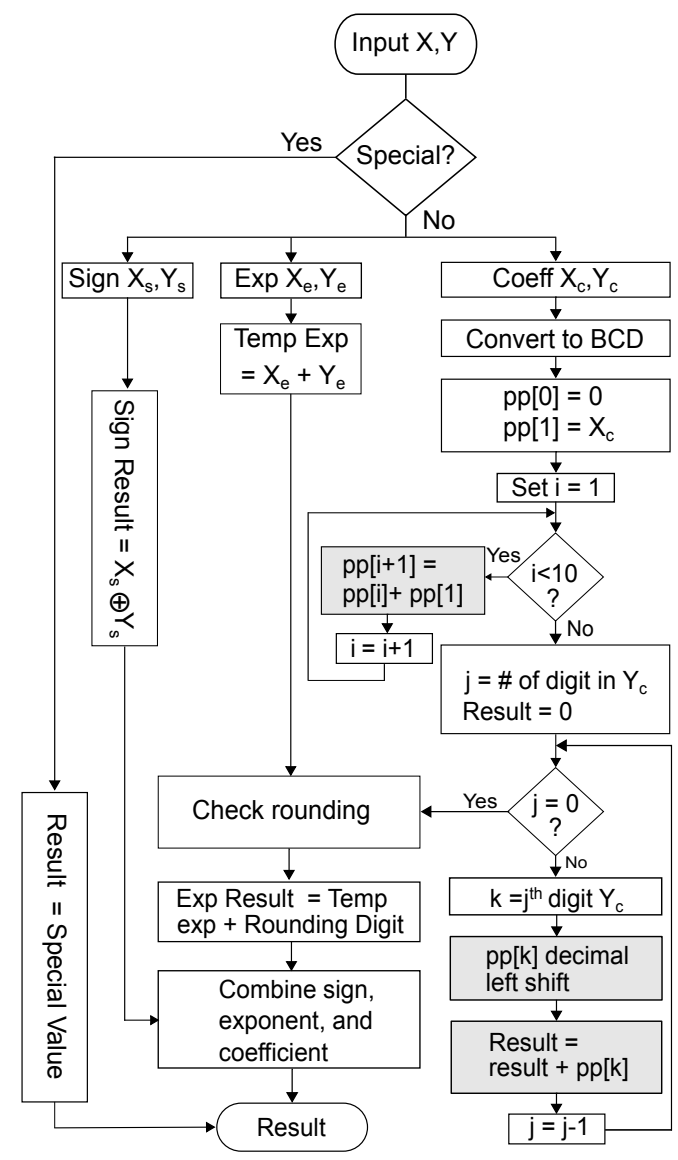

Fig. 1: Flow of method-1 in [9]. White and gray blocks for software and hardware respectively.

2) Evaluation of software-hardware co-design solution of decimal multiplication.

3) Open-source project of proposed framework available at www. decimalarith.info.

The organization of the paper is as follows: In Section II decimal multiplication using software hardware co-design are discussed. In section III the overview of the proposed framework are presented. The evaluation of decimal multiplication using the framework is proposed in Section IV and the evaluation results are discussed in V. Finally, the conclusion is provided in Section VI.

\section{Co-Design for Decimal Multiplication}

Decimal floating-point (DFP) number system represents floating-point number using base 10. A number is finite or special value. Every finite number has three integer parameters sign, coefficient, and exponent.

IEEE 754-2008 compliant decimal multiplication process has the following basic steps: first, both operands are checked whether they are a finite number or special values such as $\mathrm{NaN}$ (not a number) or infinity. If either operand is a special value, then the special general rules are applicable for the operation; otherwise, the operands are multiplied together with following basic steps:
TABLE I: Development environment

\begin{tabular}{l|l}
\hline & Description \\
\hline \hline Compiler & GNU GCC for RISC-V as a cross compiler [14] \\
\hline ISA simulator & SPIKE [15] \\
\hline Programming language & $\begin{array}{l}\text { High-level: Scale, C++, and C } \\
\text { Hardware description language: Chisel [16] } \\
\\
\text { Assembly: RISC-V assembly }\end{array}$ \\
\hline ISA & RISC-V [12] \\
\hline Processor core & Rocket [17] \\
\hline Decimal Software Library & decNumber C library [2] \\
\hline Testing & Arithmetic operations verification database [18] \\
\hline
\end{tabular}

- The sign and initial exponent are calculated by doing XOR and addition operation between the signs and exponents of multiplier and multiplicand.

- Coefficient multiplication is performed to produce the coefficient of the result.

- If the result exceeds the precision then rounding operation is applied with various rounding algorithm [4].

- Finally, the exponents are adjusted accordingly.

The process of such multiplication can be designed using a combination of software and hardware. Some solutions have been proposed in [9]. In this paper, we propose an evaluation framework and evaluate Method-1 of [9] as an example.

Figure 1 shows an overall flow of Method-1 that is one of the solutions in [9]. The method requires one BCD-CLA (BCD carry-lookahead adder) as an accelerator to generate multiplicand multiples and accumulate partial products. In addition, no binary conversion is required. To obtain the product of coefficients of multiplicand $\left(X_{c}\right)$ and multiplier $\left(Y_{c}\right)$, these values are first converted into BCD binary-coded decimal from DPD (densely packed decimal) [13] in software. The DPD encoding, where the coefficient encoding is is very close to $\mathrm{BCD}$ and can be easily converted to $\mathrm{BCD}$. Hardware component $\mathrm{BCD}$ adder is used to generate multiplicand multiples $1 X_{c}$ to $9 X_{c}$ by adding $X_{c}$ repeatedly. Then the final sum is calculated by adding and shifting the multiplicand multiples according to the digit of $Y_{c}$. The exponent of the result is finalized by adding the number of the rounded digits.

\section{OVERVIEW OF FRAMEWORK}

The proposed framework uses RISC-V ecosystem, decimal $\mathrm{C}$ library (decNumber), arithmetic verification test case and our developed set of test programs. All major components and software tools, used to integrate the framework, are listed in Table I. Note that they are fully open-source programs.

In a co-design methodology, it is very important to decide which functions or operation should have dedicated hardware, and which functions should remain in software to reduce hardware overhead and increase the speed of computing with several tradeoffs. Many parameters including encoding system (BID, DPD), internal format of a decimal number, base, etc. need to be considered for the design.

To design the framework considering software-hardware co-design, we develop a set of hardware components and software program. We include some area efficient hardware components along with associated software that supports 


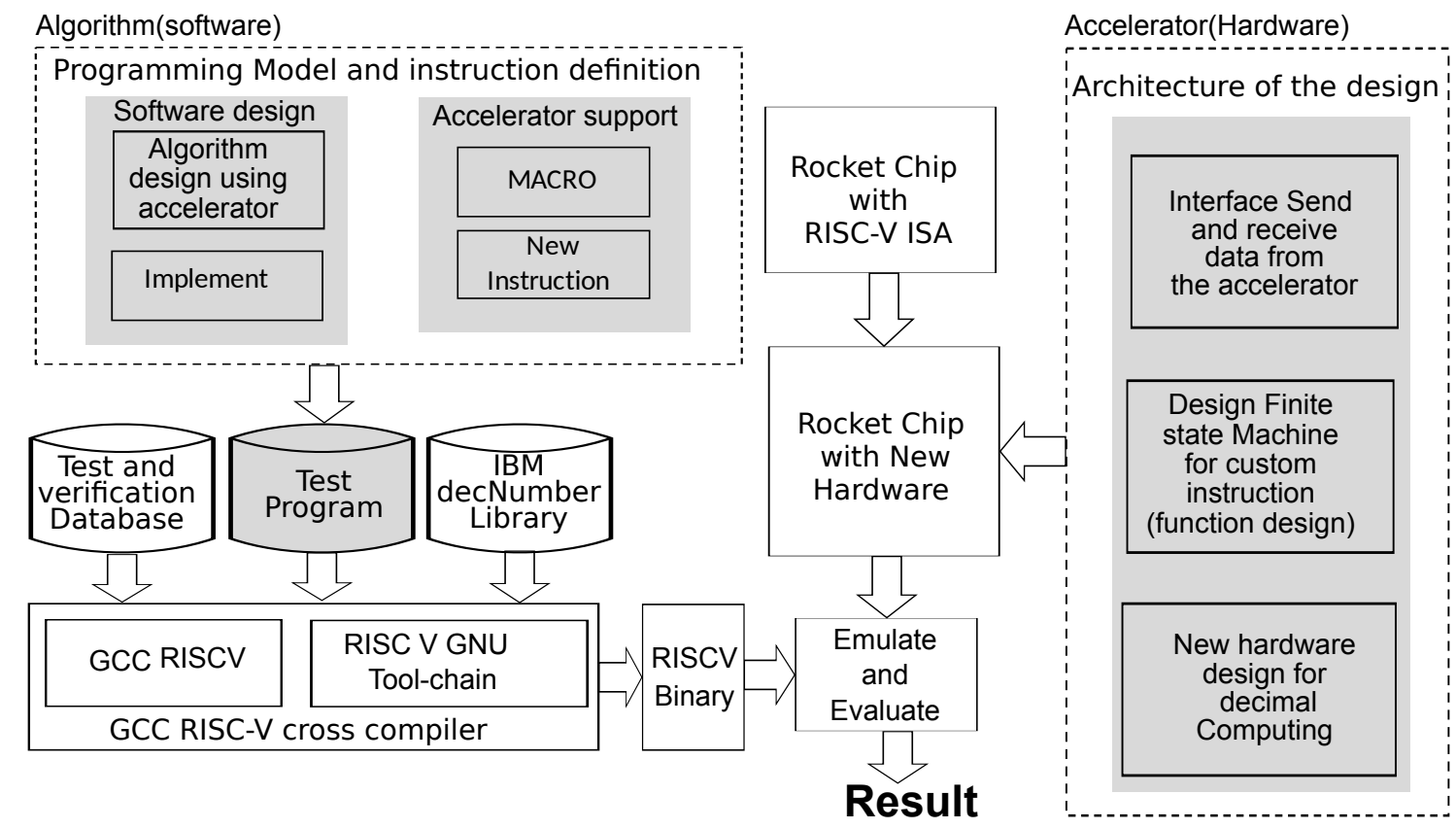

Fig. 2: Overview of proposed framework. Gray color indicates our contribution.

decimal computing. Hardware components are realized as a dedicated accelerator. RISC-V based Rocket core and Rocket custom coprocessor (RoCC) are used in the framework.

The software design may adopt some existing process form [2], [3] with replacement of some expensive and suitable portion with hardware like [9], or a completely new method with new instructions can also be designed. In our design, we use base billion, DPD encoding, with BCD-8421 on hardware. However, the design parameter can be flexibly changed by the framework user.

In addition to hardware and corresponding software, we also develop a test program generator written in C. The purpose of the generator is to configure the parameters. To use the generator, we first set up some mandatory and optional configurations including the format of precision (double or quad), input data-type (rounding, overflow, normal, underflow, etc.), type of the arithmetic operation (addition, subtraction, multiplication or any other), the number of repetition par calculation, pattern of output (execution time or number of cycle) etc. Then the test program to evaluate target co-design solution is automatically generated.

\section{EVAluation Framework}

The architecture (hardware) and programming model (software) are described in this section. The overall model of the proposed framework is depicted in Fig. 2. On the hardware part, necessary FSM and hardware description for the accelerator are designed. Rocket chip is then compiled and built with the newly generated accelerator and an executable for the emulator is generated. On the other hand, in software part, RISC-V in-line assembly and $\mathrm{C}$ source code are compiled by RISC-V GCC cross compiler to generate RISC-V binary. After that, the binaries are simulated by SPIKE ISA simulator

\begin{tabular}{|l|l|l|l|l|l|l|l|}
\hline $\begin{array}{l}\text { Function7(7) } \\
\text { rocc instruction }\end{array}$ & $r s 2(5)$ & $r s 1(5)$ & $\begin{array}{c}\text { xd } \\
\text { (1) }\end{array}$ & $\begin{array}{l}\text { (1) } \\
(1)\end{array}$ & $\begin{array}{l}\text { (1) } \\
(1)\end{array}$ & $r d(5)$ & $\begin{array}{l}\text { Opcode(7) } \\
\text { Custom 0-3 }\end{array}$ \\
\hline
\end{tabular}

Fig. 3: RoCC instruction encoding (number of bits).

TABLE II: List of instructions

\begin{tabular}{l|l|l}
\hline Function & Function7 & Description \\
\hline WR & 0000000 & $\begin{array}{l}\text { Write a value } \\
\text { to a register in Rocket core }\end{array}$ \\
\hline RD & 0000001 & $\begin{array}{l}\text { Read a value } \\
\text { from a register in Rocket core }\end{array}$ \\
\hline LD & 0000010 & Load a value from a memory \\
\hline ACCUM & 0000011 & $\begin{array}{l}\text { Accumulate a value into a register } \\
\text { in Rocket core }\end{array}$ \\
\hline DEC_CNV & 0000110 & $\begin{array}{l}\text { Convert binary number to } \\
\text { corresponding BCD }\end{array}$ \\
\hline DEC_MUL & 0000111 & Multiply two BCD numbers \\
\hline DEC_ADD & 0000100 & Add two BCD numbers \\
\hline DEC_ACCUM & 0001000 & $\begin{array}{l}\text { Accumulate BCD numbers stored } \\
\text { in internal registers }\end{array}$ \\
\hline
\end{tabular}

for functional verification. Hereafter RISC-V machine code is generated to be executed on the emulator. Finally, the emulator is executed to get the evaluation output and wave forms. A detailed description of architecture (hardware) and programming model (software) is given below:

\section{A. Architectural Design (Hardware)}

To embed the decimal arithmetics into a RoCC coprocessor, two major parts, interfacing and executing units, are required. RoCC has three default interface signals and they are:

1) Core control (CC): for co-ordination between an accelerator and Rocket core. 
TABLE III: RoCC instructions (number of bits)

\begin{tabular}{|c|c|c|c|c|c|c|c|}
\hline 31 & 25 & 19 & 15 & & & 11 & 6 \\
\hline Instruction & Source -1 & Source-2 & \multicolumn{3}{|c|}{ Addressing mode } & Destination & Fixed opcode \\
\hline $\begin{array}{l}\text { Function } 7(7) \\
\text { roce instruction }\end{array}$ & rs2(5) & rs1(5) & $\begin{array}{l}\mathrm{xd} \\
(1)\end{array}$ & $\begin{array}{l}\text { Xs1 } \\
\text { (1) }\end{array}$ & $\begin{array}{l}\text { Xs2 } \\
(1)\end{array}$ & $r d(5)$ & $\begin{array}{l}\text { Opcode(7) } \\
\text { Custom-0 }\end{array}$ \\
\hline CLR_ALL (0000101) & 00000 & 00000 & 0 & 0 & 0 & 00000 & 0010111 \\
\hline RD (0000010) & 00000 & 01011 & 0 & 0 & 1 & 00000 & 0010111 \\
\hline WR $(0000000)$ & 00000 & 01011 & 1 & 0 & 0 & 00000 & 0010111 \\
\hline DEC_ADD (0000100) & 01010 & 01011 & 1 & 1 & 1 & 01100 & 0010111 \\
\hline
\end{tabular}

2) Register mode (Core): for the exchange of data between an accelerator and Rocket core.

3) Memory mode (Mem): for communication between an accelerator and L1-D Cache.

Besides the default interface, there is some extended RoCC interface like floating-point unit for send and receive data from an FPU and few more. The interface between the core and accelerator with default interface signal that communicates through core and memory by the command $(\mathrm{cmd})$, response (resp).

Figure 3 shows RoCC 32-bit custom instruction encoding with the bit length of several parts of the instruction. The opcode is selected from custom- 0 to custom- 3 reserved for custom instructions, and each opcode realizes several functions using function 7 bits. Each instruction can have at most one destination register $r d$ and two source registers $r s 1$ and $r s 2$.

Three flags $\mathrm{xd}$, xs1, and $\mathrm{xs} 2$ specify whether registers in Rocket core are used as destination or source registers. That is, these flags show the necessity of synchronization between Rocket core and the accelerator. If the flag value is 1 , a register in Rocket core is used, that is, the values are transferred with the instruction when $\times s 1=" 1$ " or $\times s 2=" 1$ " and Rocket core waits for the response when $\mathrm{xd}=$ " 1 ", otherwise, it specifies the address of the register file in the accelerator or the corresponding field is not used.

Table II lists the developed instructions for decimal arithmetic in the framework. Though we have already developed some of instructions with dedicated hardware, any such hardware component can be integrated into the design.

The RoCC interface is used to realize communication between Rocket core and the accelerator or between memory (cache) and the accelerator through $c m d$ and resp. The accelerator receives and decodes the commands from Rocket core. Depending upon the value of function 7, the corresponding function is executed. Table III shows the list of some new instructions used in the framework with corresponding values, where core internal integer register 10 and 11 are used as source registers and 12 is used as the destination register. Figure 4 shows the high-level architecture of the accelerator, while Fig. 5 shows the implementation of the finite state machine required to implement the accelerator.

For example, when (DEC_ADD instruction is required, it performs BCD (Binary Coded Decimal) addition for two operands and writes the result to the destination register. The

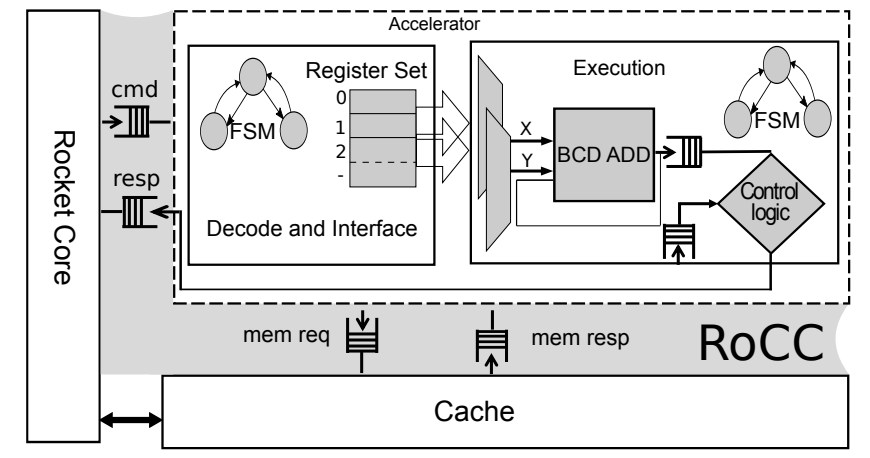

Fig. 4: Basic block of Method-1 in [9].

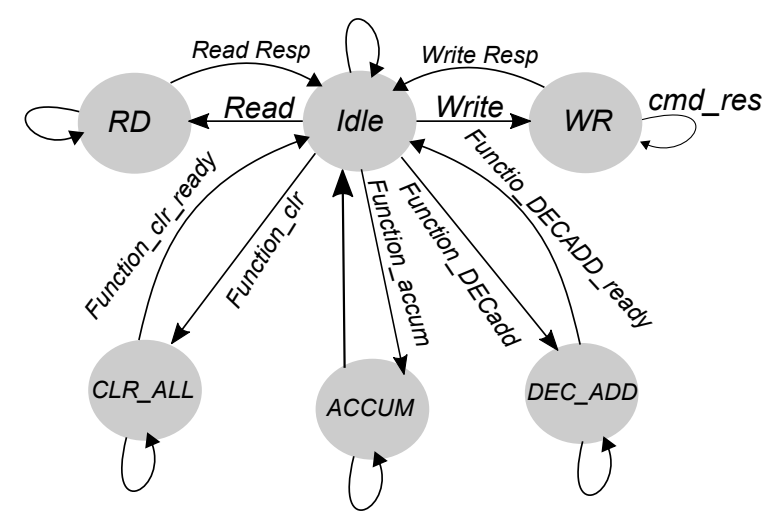

Fig. 5: Interface FSM of required function for Method-1 in [9].

pseudo-code written in Chisel for an instruction DEC_ADD (function7 = "0000100") is as follows.

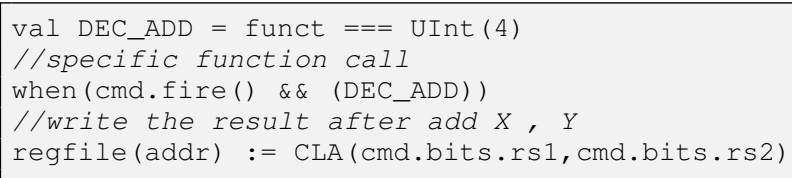

When the accelerator receives DEC_ADD command, it executes the command by adding values of two source registers rs 1 and rs 2 and then write the result to destination register xd. Here CLA (Carry-lookahead adder) performs BCD add operation. Once the accelerator design is completed, the new chip is generated with the new accelerator.

\section{B. Programming Model (Software)}

A set of software programs are implemented for the main algorithm where dedicated functions are used to call new instructions supported by the accelerator. 
TABLE IV: Average number of cycles of Method-1 using the framework

\begin{tabular}{l|r|r|r|r}
\hline & SW Part & HW part & Total & Speedup \\
\hline Method-1 [9] & 1013 & 188 & 1201 & $2.73 \mathrm{x}$ \\
\hline Software [2] & 3285 & 0 & 3285 & - \\
\hline $\begin{array}{l}\text { Method-1 using } \\
\text { dummy function [9] }\end{array}$ & 1446 & 0 & 1446 & $2.27 \mathrm{x}$ \\
\hline
\end{tabular}

TABLE V: Evaluation of Method-1 by real implementation

\begin{tabular}{l|r|r}
\hline & Time (sec) & Speedup \\
\hline $\begin{array}{l}\text { Method-1 using } \\
\text { dummy function [9] }\end{array}$ & 589 & $2.32 \mathrm{x}$ \\
\hline Software [2] & 1367 & - \\
\hline
\end{tabular}

The fragments of the pseudo-code using accelerator are as follows:

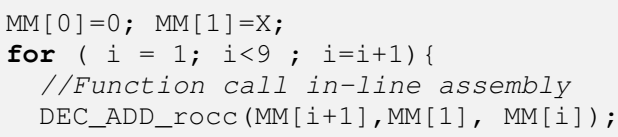

The first fragment is used to generate multiplicand multiple and the second fragment is for accumulating the partial product to generate final result (See Fig. 1 on Sec. II). $D E C \_A D D \_r o c c$ is a function to call $D E C_{-} A D D$ instruction. The function $D E C \_A D D \_r o c c$ takes two BCD inputs and return the result after $\mathrm{BCD}$ addition. A set of MACROs and inline assembly programs are used to define the function $B C D \_A D D \_r o c c$. In this example code, $\operatorname{MID}(\mathrm{A}, \mathrm{B}, \mathrm{C})$ is MACRO to extract the specific bit of A within the range $C$ to B. As both source are in BCD format, where every four bit represents one digit, thus in every cycle of the for loop pick one digit of multiplier and then add multiplicand multiple to generate the final product. The function that calls DEC_ADD instruction is as follows.

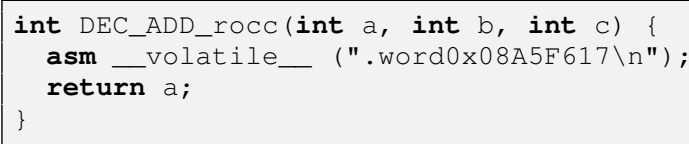

In the code, "0x08A5F617" is the hex value for instruction custom-0 (DEC_ADD), where Rocket internal registers 10 and 11 are used as source registers and 12 is used as the destination register. In our framework, we also provide a set of dynamic MACROs to automatically generate the hex value of corresponding instruction.

\section{EXPERIMENTAL RESULTS}

In [9], the performance has been evaluated by replacing the hardware part with a static function called dummy function. The dummy functions have a fixed return type and designed according to methods algorithm. This dummy functions are called from the software function repeatedly according to
TABLE VI: Evaluation of Method-1 using Gem-5 targeting RISC-V ISA

\begin{tabular}{l|r|r}
\hline & Time (sec) & Speedup \\
\hline $\begin{array}{l}\text { Method-1 using } \\
\text { dummy function [9] }\end{array}$ & 0.005443 & $2.30 \mathrm{x}$ \\
\hline Software [2] & 0.012511 & - \\
\hline
\end{tabular}

method design. This approach is considered to include interfacing time for software and hardware. Such an evaluation process has the following limitation:

1) Computing time highly dependent on the nature of the input, like rounding operation takes higher time than normal operation. However, the dummy function always return a fixed value and the execution may not follow the expected flow.

2) The cycle time for the processor may not the same with and without new hardware.

Method-1 of [9] with hardware accelerator is implemented and evaluated total number of cycles with 8,000 sample inputs including overflow, underflow, normal, rounding, and clamping cases. For comparison, IBM decNumber C library for double precision decimal floating-point arithmetic [2] is examined as a software-based solution, and Method-1 with dummy functions is also examined. We use RISC-V RDCYCLE instruction to count the number of cycles. Table IV summarizes the result. From the result, it is shown that Method-1 with the accerlator is 2.73 times faster than the software-based solution, while Method-1 with dummy functions is 2.27 times faster.

The result of Table IV is now compared with two other evaluations to compare a software-based solution (IBM decNumber C library) and Method-1 with dummy functions. In Table $\mathrm{V}$, real implementations of these two methods are executed at Windows 10, 64-bit on Intel core i7 $2.29 \mathrm{GHz}$ with 8 GB RAM. The second evaluation uses Gem-5 [19] simulator with AtomicSimpleCPU at system call emulation (SE) mode. In SE mode we need to specify a binary file to be executed of Method-1 of [9]. In this evaluation, we use RISC$\mathrm{V}$ as the target ISA [12]. Here we also use 8,000 sample and the result is summarizes in Table VI. From Table IV through Table VI, it is found that dummy function based evaluations in three different evaluation exhibit almost the same speedup (2.27x, 2.32x, 2.30x). That shows our proposed framework accurately evaluated the cycle times for the target program. The proposed framework shows the exact result with 2.73 times improvement in cycle times. The outcome of the result presents a successful evaluation of the framework. Our proposed framework design considering hardware accelerator. Such an interface imposes a latency overhead during data exchange with CPU because of the position of the interface into the pipeline. It also depends on how the core and interface are handled. The impact of such a problem depends upon the frequency of data exchange between the main core and the accelerator. Also, due to cache random replacement policy, Rocket chip is responsible for computing the number of cycles in nondeterministically. However, as the framework is 
proposed for the integrated evaluation where a large numbers of input samples with many repetition, the framework can show statistically meaningful results.

\section{CONCLUSION}

This paper presents an integrated evaluation framework using the RISC-V ecosystem, IBM decNumber library, verification test database with our developed set of test programs. he framework is designed to accurately evaluate software-hardware co-design based decimal computation. A decimal multiplication based on software hardware co-design is implemented to the framework to validate the concept of combined decimal multiplication by the actual result. The framework can perform both functional and behavioral evaluation of any such software-hardware co-design of decimal computation. The framework is an open-source project, and links to all of the source files are available online at www. decimalarith.info.

\section{REFERENCES}

[1] "IEEE standard for floating-point arithmetic IEEE Std 754-2008," pp. $1-70,2008$.

[2] "C decNumber Library : access date 2018-01-02," [Online: http:// speleotrove.com/decimal/decnumber.html].

[3] "IEEE 754-2008 Decimal Floating-Point Compliance Library," [Online: https://software.intel.com/en-us/articles/ intel-decimal-floating-point-math-library].

[4] M. Cornea, J. Harrison, C. Anderson, and P. T. P. Tang, "A software implementation of the IEEE 754R decimal floating-point arithmetic using the binary encoding format," IEEE Transactions on Computers, vol. 58, no. 11, pp. 148-162, 2009.

[5] S. Carlough, A. Collura, S. Mueller, and M. Kroener, "The IBM zEnterprise-196 decimal floating-point accelerator," in Proceedings of IEEE International Symposium on Computer Arithmetic, 2011, pp. 139146.

[6] E. M. Schwarz, J. S. Kaepernick, and M. F. Cowlishaw, "The IBM z900 decimal arithmetic unit," in Asilomar Conference on Signals Systems and Computers, 2001, pp. 1335-1339.

[7] E. M. Schwarz, J. S. Kapernick, and M. F. Cowlishaw, "Decimal floating-point support on the IBM system z10 processor," IBM Journal of Research and Development, vol. 53, no. 1, pp. 4.1-4.10, 2009.

[8] "Fujitsu's new generation SPARC64 processor," [Online: http://www.fujitsu.com/global/products/computing/servers/unix/ sparc-enterprise/key-reports/featurestory/sparce-feature1209.html].

[9] M. R. ul haque, M. Shintani, and M. Inoue, "Decimal multiplication using combination of software and hardware," in Proceedings of IEEE Asia Pacific Conference on Circuits and Systems, 2018, pp. 239-242.

[10] "OpenSPARC T1,T2 processor design source code, simulation tools, design verification suites, and other tools under open-source licenses," [Online: http://www.opensparc.net].

[11] "Oracle SPARC Architecture," [Online: https://www.oracle.com/ technetwork/sparc-architecture-2015-2868130.pdf].

[12] "The RISC-V Instruction Set Manual, Volume I: Base User-Level ISA," [Online: https://riscv.org/].

[13] M. Cowlishaw, "Densely packed decimal encoding," IEE Proceedings - Computers and Digital Techniques, vol. 149, pp. 102-104, 2002.

[14] "GCC, the GNU Compiler Collection," [Online: https://gcc.gnu.org/].

[15] "Spike RISC-V ISA Simulator," [Online: https://github.com/riscv/ riscv-isa-sim]

[16] J. Bachrach, H. Vo, B. Richards, Y. Lee, and A. Waterman, "Chisel: constructing hardware in a scala embedded language," in Proceedings of IEEE/ACM Design Automation Conference, 2012, pp. 465-471.

[17] “The Rocket Chip Generator," [Online: https://riscv.org].

[18] A. A. R. Sayed-Ahmed, H. A. H. Fahmy, and M. Hassan, "Three engines to solve verification constraints of decimal floating-point operations," in Asilomar Conference on Signals Systems and Computers, 2010, pp. 1-4.
[19] N. Binkert, B. Beckmann, G. Black, S. K. Reinhardt, A. Saidi, A. Basu, J. Hestness, D. R. Hower, T. Krishna, S. Sardashti, R. Sen, K. Sewell, M. Shoaib, N. Vaish, M. D. Hill, and D. A. Wood, "The Gem5 simulator," ACM SIGARCH Computer Architecture News, vol. 39, no. 2 , pp. 1-7, 2011. 